\title{
Cesárea electiva en paciente portadora de procedimiento de Fontan
}

\section{Elective caesarean section in a patient with the Fontan procedure}

María Laura Illescas, Renzo García², Roberto Puñales³ , Juan Pablo Bouchacourt, Juan Antonio Riva 5

\begin{abstract}
The development of a single ventricle circulation palliated with the Fontan operation has determined that an increasing number of women complete their pregnancy successful. The physiological changes of pregnancy mean a high demand on the cardiovascular system which has high dependence on preload and fixed stroke volume. The risk of cardiovascular complications and maternal and fetal mortality is higher. We report the first case registered in Uruguay of a patient with Fontan circulation who during the third trimester presented persistent hypoxemia without heart failure. The route of delivery was an elective caesarean section because of fetal indication. This was performed under epidural anesthesia without major incidents. Maternal and fetal outcome were successful.
\end{abstract}

\section{RESUMEN}

La cirugía de Fontan para pacientes con ventrículo único a determinado que un número creciente de mujeres completen su gestación de forma segu-

\section{Key words:}

Fontan palliation, pregnancy, anesthesia, single ventricle congenital heart disease

1 Médico Anestesióloga. M.D. Profesora Adjunta Departamento de Anestesiología. Facultad de Medicina. Universidad de la República. Uruguay.

2 Médico General. M.D. Residente Departamento de Anestesiología. Facultad de Medicina. Universidad de la República. Uruguay.

3 Médico Anestesiólogo. M.D. Asistente Departamento de Anestesiología Facultad de Medicina. Universidad de la República. Uruguay.

4 Médico Anestesiólogo. PhD. Profesor Agregado Departamento de Anestesiología Facultad de Medicina. Universidad de la República. Uruguay.

5 Médico Anestesiólogo. M.D. Profesor Director Departamento de Anestesiología. Facultad de Medicina. Universidad de la República. Uruguay.

Fecha de recepción: 10 de junio de 2020

Fecha de aceptación: 14 de julio de 2020

\section{ORCID}

0000-0001-5800-3181

\section{Correspondencia:}

Dra. Laura Illescas

laura.illescas@gmail.com 
ra. Los cambios fisiológicos del embarazo pueden resultar deletéreos dada la dependencia de precarga con imposibilidad de aumentar el volumen sistólico. Determinando un mayor riesgo de complicaciones y mortalidad maternofetal. Presentamos el primer caso registrado en Uruguay de una paciente portadora de circulación de Fontan que durante el tercer trimestre presenta hipoxemia persistente sin falla cardíaca realizándose una cesárea electiva por indicación fetal. Se realizó una anestesia peridural sin complicaciones. Los resultados materno-fetales fueron satisfactorios.

\section{Palabras clave:}

Circulación de Fontan, embarazo, anestesia, ventrículo único, cardiopatía congénita

\section{Introducción}

D ebido al desarrollo de la cirugía cardiaca en cardiopatías congénitas (CC), mayor número de pacientes portadoras de circulación de Fontan llegan a completar un embarazo. Los partos en mujeres con CC rondan el 34,9\%[1]. Las portadoras de CC complejas tienen mayor posibilidad de morbimortalidad materno-fetales 2,3. El ventrículo único (VU) corresponde al 0,05/1.000 de ellas, siendo la corrección de Fontan la técnica más frecuente para repararla en forma paliativa[1]. Los cambios del embarazo pueden resultar deletéreos dada la dependencia de la precarga y la imposibilidad de aumentar el volumen sistólico del VU[4],[5].

Presentamos una paciente con corrección de Fontan con tubo intra-cardíaco que en el tercer trimestre del embarazo se decidió la interrupción por vía cesárea dado el contexto de hipoxemia materna que podría comprometer el pronóstico perinatal.

Hasta donde sabemos este es el primer caso de embarazo exitoso en una paciente portadora de circulación de Fontan en Uruguay.

\section{Caso clínico}

MB. 27 años, embarazo de 34 semanas, mal tolerado por diabetes gestacional.

Portadora de VU con corrección de Fontan. Inicialmente se le realizó cirugía de derivación cavopulmonar bidireccional (cirugía de Glenn) con resección de septum interauricular, completándose con procedimiento de Fontan, tubo intracardíaco y fenestra sin cierre posterior.

Buena evolución con aceptable clase funcional, sin arritmias, falla cardiaca ni eventos tromboembólicos, tratada con acido acetilsalicílico (AAS).

Embarazo no programado, ingresa a centro terciario a la semana 18 de gestación por hipoxemia. Ecocardiograma: VU de doble entrada, presiones bajas en el sistema, buena función ventricular, FEVI $58 \%$. Electrocardiograma: normal. Test de la Marcha: recorre 88 metros desaturando a $84 \%$ con $\mathrm{FiO}_{2} 60 \%$. $\mathrm{SatO}_{2}$ de $84 \%-85 \%$ aumentando a $90 \%-91 \%$ con $\mathrm{FiO}_{2} 28 \%$. Pro-BNP: $410 \mathrm{pg} / \mathrm{ml}$.

Tratada con heparina de bajo peso molecular (HBPM) $40 \mathrm{mg} / \mathrm{día}$ y AAS $100 \mathrm{mg} / \mathrm{día}$. Carvedilol 25 $\mathrm{mg} /$ día y Furosemide $40 \mathrm{mg} / \mathrm{día}$ en el primer trimestre.

Dado riesgo de injuria neurológica fetal por hipoxia materna, se decide interrumpir el embarazo.

Seleccionamos una técnica peridural con monitorización estándar. La paciente se negó a la colocación del catéter arterial. Administramos cristaloides: $500 \mathrm{ml}$ i/v y posteriormente por vía peridural, Bupivacaina al 0,5\% $118 \mathrm{mg}$ y Fentanilo $50 \mathrm{mcg}$ hasta un nivel sensitivo T4. No presentó alteraciones hemodinámicas. $\mathrm{SaO}_{2}: 85 \%-87 \%$. Utilizamos carbetocina como uterotonico sin alteraciones hemodinámicas. Recién nacido vivo, APGAR 7/9, requirió cirugía por vólvulo intestinal evolucionando favorablemente.

Cursó 72 h en la unidad de Recuperación Postanestésica. A las $24 \mathrm{~h}$ oligoanuria y edemas de miembros inferiores hasta raíz de muslo. Sin otros elementos de insuficiencia cardíaca. Se trató con diuréticos de asa, con respuesta óptima. Se reanudó HBPM y carvedilol. ProBNP 247 pg/ml. Buena evolución, asintomática a los 3 meses.

\section{Discusión}

El porcentaje de partos en pacientes con CC es bajo $(0,07 \%)$, presentan mayor mortalidad, posibilidad de eventos cardíacos (arritmias, falla cardíaca)[6], mayor número de cesáreas, retardo en el crecimiento y muerte fetal[2],[3]. Las pacientes con circulación de Fontan presentan una alta incidencia de complicaciones maternas: arritmias, falla cardíaca y eventos tromboembólicos (11\%-25\%)[4],[5],[6].

El procedimiento de Fontan conecta el flujo cava a la circulación pulmonar, evitando el ventrículo, ge- 
nerando flujo sanguíneo pasivo con riesgo de tromboembolismo, arritmias y falla cardíaca[6],[7]. En esta paciente el procedimiento se realizó, primero conectando la vena cava superior a la arteria pulmonar y posteriormente conectando el resto del flujo venoso con un tubo intracardíaco. Este tubo favorece la aparición de arritmias, tromboembolismo y altera la función del ventrículo sistémico. Actualmente, la tendencia es utilizar un tubo simple extracardíaco, realizando conexiones cavo pulmonares y evitando la anastomosis aurículo-pulmonar.

La paciente no presentó complicaciones. Llegó al tercer trimestre con una situación hemodinámica estable y un feto viable. Durante este período se espera una disminución marcada de las resistencias vasculares y aumento del volumen plasmático (30\%-50\%). Por lo cual, el gasto cardíaco debería incrementarse $50 \%$, situación difícil de sobrellevar en estas condiciones, siendo altamente dependiente de la precarga con muy poca capacidad de aumentar el volumen sistólico[4],[8]. A pesar de esto, la mayoría de las pacientes que llegan al tercer trimestre lo hacen con aceptable clase funcional y FEVI conservada[5]. Los factores propuestos como predictores de eventos cardíacos durante el embarazo son: eventos cardíacos previos, clase funcional mayor a II (NYHA) y/o cianosis, obstrucción del ventrículo izquierdo o FEVI menor a 40\%[9]. Otros autores plantean que la evaluación cardiológica para determinar el riesgo debería incluir las medidas a nivel de la circulación de Fontan, ya que la buena función ventricular no es predictivo de buena evolución materno-fetal[11].

La decisión de la interrupción del embarazo se fundamentó en la persistente hipoxemia que podría poner en riesgo al feto. Su efecto sobre la implantación embrionaria a sido planteado como principal causa de aborto espontáneo (40\%)[4],[8]. No hay suficiente evidencia sobre lo que sucede con el desarrollo neonatal, pudiendo generar un entorno adverso para el desarrollo neurológico. La indicación de cesárea, ante la ausencia de una clara recomendación, se ha basado en la creencia que esta vía tendría menores efectos en la circulación de Fontan, sin embargo no hay evidencia que lo sustente. En el parto vaginal las perdidas sanguíneas son menores, especialmente en ausencia de elementos de falla cardíaca severa o condiciones obstétricas que indiquen una cesárea. Se propone realizar una prueba de parto con colocación de catéter peridural para analgesia y asistencia con forceps del período expulsivo[11]. En varias publicaciones[11], la mayoría de los nacimientos fueron partos vaginales, y las cesáreas fueron indicadas por compromiso fetal. En otra serie, más del $50 \%$ de los nacimientos fueron por cesárea[6]. Las causas serían: fetos pequeños para la edad gestacional, pretérminos y falta de experiencia con estas pacientes[3],[5],[8].

Existe coincidencia en utilizar bloqueos neuroaxiales para partos y cesáreas, evitando la anestesia general con ventilación mecánica por las repercusiones sobre el retorno venoso pasivo. La presión negativa intratorácica es determinante del retorno venoso adecuado, mientras que la presión positiva disminuirá el flujo sanguíneo pulmonar y el gasto cardíaco[11]. Optamos por una anestesia peridural con un bloqueo progresivo y por tanto una mejor adaptación hemodinámica[5],[11]. Dada la dependencia de la precarga por un flujo pasivo, es importante evitar la deshidratación[11], por lo cual, se administró cristaliodes previo al bloqueo.

Es necesaria la monitorización invasiva de la presión arterial y venosa para la detección rápida de los cambios hemodinámicos[11], sin embargo, esto fue descrito para casos de cesáreas urgentes. Esta decisión debe ser tomada de acuerdo al estado funcional cardíaco previo a la cirugía[7]. Dado la negativa de la paciente, su condición clínica y siendo una cesárea electiva, realizamos monitorización no invasiva.

Presentamos el caso de una paciente con cirugía paliativa de Fontan con tubo intracardíaco, que cursa un embarazo no planificado en el tercer trimestre sin complicaciones cardiovasculares. Debido a la hipoxemia se decide interrumpir el embarazo en forma electiva. Se planificó una adecuada reposición previa, monitorización estándar y anestesia peridural fraccionada. No hubo eventos cardíacos y tanto la paciente como su hija evolucionaron favorablemente en su domicilio tres meses después del procedimiento.

\section{Conclusión}

Nos enfrentaremos con mayor frecuencia a pacientes portadoras de circulación de Fontan que llevarán adelante una gestación. A pesar del elevado riesgo de complicaciones y muerte materno-fetales, un seguimiento con la participación del anestesiólogo en la toma de decisiones permitirá diseñar una estrategia reduciendo en forma significativa estos riesgo. Para ello el anestesiólogo debe estar adecuadamente preparado.

\section{Reconocimientos}

Agradecemos a todo el equipo asistencial por su trabajo logrando un resultado exitoso. También queremos agradecer a nuestra paciente quien brindó su consentimiento para la realización de esta publicación. 


\section{Referencias}

1. Marelli AJ, lonescu-ittu R, Mackie AS GL, M. ND and K. Lifetime Prevalence of Congenital Heart Disease in the General Population from 2000 to 2010. Circulation. 2014;130(9):749756. https://doi.org/10.1161/ CIRCULATIONAHA.113.008396

2. Schlichting LE, Insaf TZ, Zaidi AN, Lui GK VZA. Maternal Comorbidities and Complications of Delivery in Pregnant Women With Congenital Heart Disease. JACC. 2019;73(17):21812191. https://doi.org/10.1016/j. jacc.2019.01.069

3. Hayward RM FE and TZ. Maternal and Fetal Outcomes of Admission for Delivery in Women With Congenital Heart Disease. JAMA Cardiol. 2017;2(6):664671. https://doi.org/10.1001/ jamacardio.2017.0283

4. Rychik J, Atz AM, Celermajer DS, Deal BJ, Gatzoulis MA GM et al. Evaluation and Management of the Child and Adult with
Fontan Circulation. Circulation. 2019:324-384. Circulation. 2019; CIR0000000000000696. https://doi.org/10.1161/ CIR.0000000000000696

5. Bonner SJ, Asghar O, Roberts A, Vause S, Clarke B, Keavney B. Cardiovascular, obstetric and neonatal outcomes in women with previous fontan repair. Eur J Obstet Gynecol Reprod Biol. 2017;219:53-56. https://doi.org/10.1016/j.ejogrb.2017.10.013

6. García Ropero A, Baskar S, Roos Hesselink JW, Girnius A, Zentner D SL and cols. Pregnancy in Women With a Fontan Circulation A Systematic Review of the Literature Downloaded. Circ Cardiovasc Qual Outcomes. 2018;11:111. https://doi.org/10.1161/ CIRCOUTCOMES.117.004575

7. Tiouririne $M$, de Souza DG, Beers KT, Yemen TA. Anesthetic Management of Parturients With a Fontan Circulation: A Review of Published Case Reports. Semin Cardiothorac Vasc Anesth.
2015;19(3):203-209. https://doi. rg/10.1177/1089253214566887

8. Cohen AM, Mulvein J. Obstetric anaesthetic management in a patient with the Fontan circulation. Br J Anaesth. 1994;73(2):252-255. https://doi. org/10.1093/bja/73.2.252

9. EH, Jooste, Haft $A H$, Warwick AA, Sherman FS VM. Anesthetic care of parturients with single ventricle physiology. J Clin Anesth. 2013;25:417-423. https://doi.org/10.1016/j.jclinane.2012.08.003

10. Ordoñez MV TJ and CSL. Success in a Fontan pregnancy : how important is ventricular function? Cardiol Young. 2018:13. https://doi.org/10.1017/ S1047951118001865

11. Monteiro RS, Dob DP, Cauldwell MR GM. Case report. Anaesthestic management of parturients with univentricular congenital heart disease and the Fontan operation. Int J Obs Anesth. 2016;28:83-91. https://doi. org/10.1016/j.ijoa.2016.08.004 